\title{
¿CÓMO PIENSA UN ACADÉMICO QUE SE HACE UNA LEY Y CÓMO SE HACE UNA LEY REALMENTE? REFLEXIONES A PARTIR DE LA INTRAHISTORIA DE LA LEY ORGÁNICA DE EDUCACIÓN (LOE) DE 2006
}

\author{
How does a scholar think an act is made and how is it actually \\ done? Some reflections based on the intra-history of the Spanish \\ Law of Education (LOE) of 2006
}

\author{
Alejandro Tiana Ferrer* \\ Fecha de recepción: 05/10/2015 • Fecha de aceptación: 12/10/2015
}

Resumen. El artículo plantea una pregunta que los historiadores de la educación se han hecho bastantes veces acerca de las conexiones y las posibles discrepancias que pueden identificarse entre la práctica política y su análisis académico. Una cosa es estudiar los procesos de elaboración y aprobación de las leyes educativas adoptando una mirada analítica externa y otra distinta adentrarse en la intrahistoria de dichas leyes. La primera posición concede la ventaja de la objetividad, pero impide en ocasiones conocer todas las variables que han jugado en los procesos de construcción de políticas. Con la intención de dar respuesta a dicha pregunta, el artículo pretende contrastar las imágenes e interpretaciones que subyacen en muchos trabajos académicos dedicados al análisis de políticas con la experiencia vivida por los actores de dichos procesos. El autor aprovecha su experiencia personal para reflexionar acerca de la tensión que suele existir entre la perspectiva interna y la externa en los procesos de cambio en educación. El trabajo está centrado en la intrahistoria de la Ley Orgánica de Educación (LOE), aportando claves para facilitar su análisis académico. Se pasa revista al proceso de preparación de la ley, a su debate, su elaboración, al proceso de su negociación y finalmente a su tramitación parlamentaria. Se exponen un conjunto de reflexiones que pueden ayudar a los lectores a combinar la mirada externa y la interna en el análisis de políticas de la educación.

Palabras clave: Política de la educación. Legislación educativa. Intrahistoria. Ley Orgánica de Educación (LOE).

* Universidad Nacional de Educación a Distancia. UNED. Facultad de Educación. Departamento de Historia de la Educación y Educación Comparada Juan del Rosal, 14. 28040 Madrid. España. atiana@edu.uned.es

Cómo citar este artículo: Tiana Ferrer, Alejandro. «¿Cómo piensa un académico que ese hace una ley y cómo se hace una ley realmente? Reflexiones a partir de la intrahistoria de la Ley Orgánica de Educación (LOE) de 2006», Historia y Memoria de la Educación, 3 (2016): 71-97. 
Abstract. The article raises a question frequently addressed by historians of education about the connections and possible discrepancies between political practice and its academic analysis. A researcher may study the processes of making and passing an educational law or, alternatively, may delve into its internal history. The first position favours an objective view, but sometimes makes it difficult to know all of the variables playing a role in the policy-making process. For the purpose of addressing such questions this paper sets out to contrast the images and interpretations underlying academic work with the experience of the actors involved in such processes. The author, relying upon his personal experience in policy-making, reflects upon the tensions between internal and external perspectives that arise when analyzing processes of educational change. The article deals with the internal history of the Law of Education (LOE), providing some keys for facilitating its academic analysis. The processes of preparation, debate, drafting, negotiation and parliamentary discussion of the law are presented and commented on. A number of reflections are included with the aim of helping readers and researchers make the best mixed use of external and internal perspectives in analyzing education policy-making.

Keywords: Education policy. Education legislation. Internal history. Law of Education (LOE).

\section{INTRODUCCIÓN}

Este artículo no es un texto académico al uso. Habiendo estado personalmente implicado en la elaboración, debate y aprobación de la Ley Orgánica de Educación (LOE), pretendo basarme en la experiencia adquirida a lo largo de ese proceso para reflexionar acerca de las conexiones y discrepancias existentes entre la práctica política y su análisis académico. ${ }^{1}$ Es mi intención contrastar las imágenes y las interpretaciones que subyacen en muchos de los trabajos que se dedican a estudiar los procesos de elaboración de las leyes educativas con la experiencia vivida personalmente. Si bien el artículo se centra en un proceso singular de elaboración de una ley determinada, me propongo trascender las circunstancias concretas de este caso y reflexionar acerca de la tensión que suele existir entre la perspectiva interna y la externa en los procesos de cambio en educación. Con ese propósito, dedicaré una atención especial a la intrahistoria de la LOE, pero procurando contrapo-

\footnotetext{
${ }^{1}$ Este artículo puede considerarse el complemento de mi libro Por qué hicimos la Ley Orgánica de Educación (Las Rozas: Wolters Kluwer, 2009) y muy especialmente de su parte quinta, titulada «Testimonio desde una cierta distancia». A él remito a los lectores para completar algunos de los aspectos aquí tratados.
} 
nerla con la mirada del observador externo. Soy plenamente consciente de que la información procedente de los participantes (como es mi caso) en un proceso como el que aquí se aborda debe ser tratada con cautela y por lo tanto invito a los lectores a contrastarla con otros análisis y testimonios, algunos de los cuales serán citados en estas páginas.

\section{LA COMPLEJIDAD DE LAS PRIMERAS DECISIONES}

Aunque muchas veces tendamos a interpretar los procesos que conducen a la aprobación de una nueva ley educativa como la aplicación de planes racionales y de estrategias bien planificadas, lo cierto es que no siempre comienzan con una idea perfectamente clara acerca de cuáles debieran ser el resultado final y el camino a seguir para alcanzarlo. Al menos, eso es lo que sucedió en el caso de la LOE.

Cuando llegué a la Secretaría General de Educación del Ministerio de Educación y Ciencia, en abril de 2004, en el equipo ministerial teníamos claro que había que dar pasos rápidos para impedir la plena aplicación de la Ley Orgánica de Calidad de la Educación (LOCE), aprobada en 2002, muchas de cuyas disposiciones debían entrar en vigor al comienzo del curso 2004-2005. Era una meta clara para todos, aunque no se hubiese planificado de antemano una estrategia concreta para alcanzarla.

Conviene recordar que la LOCE se había aprobado con una fuerte contestación, social y política, si bien la mayoría absoluta de que disponía el Partido Popular en el Congreso y el Senado le permitió sacar adelante su proyecto sin grandes dificultades ni obligación de buscar acuerdos. Al igual que sucedería con la reciente Ley Orgánica para la Mejora de la Calidad Educativa (LOMCE), apenas consiguió sumar apoyos parlamentarios, limitándose a conseguir algunas abstenciones de otros grupos políticos. Por lo tanto, se trataba de una ley contestada, cuya modificación o derogación habían planteado diversos partidos políticos de la oposición.

Debido a esa profunda discrepancia, el Partido Socialista Obrero Español (PSOE) había incluido en su programa para las elecciones generales de 2004 un epígrafe titulado "La educación, nuestra prioridad básica», que contenía un conjunto de propuestas que implicaban de hecho revertir muchas de las medidas previstas en la LOCE. Sobre esta ley el programa afirmaba que 
supone una verdadera contrarreforma de graves consecuencias educativas, personales y sociales pues segrega a los alumnos con dificultades, no prevé medidas eficaces y realistas de apoyo a los que tienen necesidades específicas; siembra de obstáculos la progresión escolar; conculca la participación de los distintos sectores de la comunidad educativa minimizando el papel de los Consejos Escolares; recupera la religión como asignatura del currículo a todos los efectos; $\mathrm{y}$, de nuevo, carece de previsiones financieras. ${ }^{2}$

En consecuencia, la tarea más urgente del nuevo equipo del Ministerio de Educación y Ciencia consistía en frenar el inmediato proceso de aplicación de la LOCE. En las primeras semanas, casi en los primeros días de legislatura, hubo que llevar a cabo un análisis detenido de la situación, con objeto de decidir cuál era la mejor estrategia a seguir.

Dos eran las opciones posibles de actuación que estuvimos barajando. La primera consistía en promover un decreto ley que revirtiese los aspectos más problemáticos de la LOCE. La ventaja de este procedimiento residía en su rapidez, pues bastaría con una decisión del Gobierno, sin perjuicio de que fuese posteriormente necesaria su convalidación parlamentaria. Los inconvenientes que dicha opción planteaba eran de dos tipos: jurídico, pues la técnica del decreto ley no es aplicable a las leyes orgánicas, como era el caso de una buena parte de los artículos controvertidos de la LOCE; y político, pues el Gobierno no quería recurrir a un instrumento de ese tipo, que a fin de cuentas hurtaría el debate legislativo de su espacio y procedimientos normales y deseables. La segunda opción consistía en retrasar las fechas de aplicación de la nueva ordenación académica, con objeto de ganar tiempo para revisar más a fondo la normativa legal vigente. La ventaja de este procedimiento consistía en que bastaba con cambiar el real decreto que regulaba el calendario de aplicación de la LOCE, ${ }^{3}$ retrasando su plena aplicación al final de los cinco años establecidos en el texto legal. Su inconveniente radicaba en la urgencia de proceder a tal cambio antes de que comenzase el curso siguiente.

\footnotetext{
2 PSOE, Merecemos una España mejor. Programa electoral. Elecciones generales 2004, pp. 163-190, referencia en p. 166, en http://www.psoe.es/source-media/000000550500/000000550997.pdf (consultado el 30 de mayo de 2015).

${ }^{3}$ Real Decreto 827/2003, de 27 de junio, por el que se establece el calendario de aplicación de la nueva ordenación del sistema educativo, establecida por la Ley Orgánica 10/2002, de 23 de diciembre, de Calidad de la Educación (BOE del 28 de junio).
} 
Aunque había poco tiempo para tomar una decisión, tuvimos que analizar cuidadosamente las opciones mencionadas, con sus respectivas implicaciones y sus condicionantes. Dado que aún no estaban totalmente constituidos los nuevos equipos del ministerio y de las secretarías correspondientes, hubo que recurrir al consejo de personas externas, generalmente compañeros de profesión y expertos cercanos, que colaboraron desinteresadamente. En pocas semanas adoptamos la decisión de elaborar un real decreto de modificación del calendario, realizamos las consultas preceptivas a la Conferencia sectorial de Educación y al Consejo Escolar del Estado, se recabó el informe del Consejo de Estado y de los diversos ministerios implicados, y finalmente se aprobó y publicó la nueva norma que venía a modificar la de 2003. ${ }^{4}$ Todo ello, en un tiempo record de siete semanas. De ese modo, se retrasaba la aplicación de los aspectos más polémicos de la LOCE y se abría una nueva etapa de trabajo. ${ }^{5}$

Conviene aludir aquí a un asunto que a menudo resulta infravalorado en los estudios de política educativa y que se refiere a la constitución y el funcionamiento de los equipos responsables de concebir, desarrollar y llevar adelante las políticas adoptadas. Acabo de señalar que el proceso se inició en este caso con unos equipos incompletos, en los que faltaban algunas piezas clave y en los que aún no se había procedido a realizar determinadas sustituciones ya decididas. Los ritmos administrativos chocaron en esta primera etapa con la urgencia política y, aunque la contradicción se resolvió más que dignamente, no dejó de generar algunos problemas.

Es muy frecuente que al estudiar las leyes educativas prestemos especial atención a quienes han sido sus promotores y sus principales actores, lo que sin duda resulta imprescindible. Pero mi experiencia me dice que no podemos quedarnos en el estudio de esos niveles más visibles, pues los procesos de construcción de políticas educativas se basan en un conjunto de aportaciones personales que desbordan esa mirada tan limitada. Creo que esto es lo que sucede, al menos en los procesos actuales, en que existe

\footnotetext{
${ }^{4}$ Real Decreto1318/2004, de 28 de mayo, por el que se modifica el Real Decreto 827/2003, de 27 de junio, por el que se establece el calendario de aplicación de la nueva ordenación del sistema educativo, establecida por la Ley Orgánica 10/2002, de 23 de diciembre, de Calidad de la Educación (BOE del 28 de mayo).

${ }^{5}$ El resultado de este proceso fue ciertamente exitoso, puesto que incluso superó varios recursos contencioso-administrativos interpuestos contra la norma. Tampoco el Consejo de Estado encontró problemas en el proyecto de real decreto que tuvo que informar preceptivamente.
} 
una complejidad administrativa y unos instrumentos normativos bastante sofisticados que soportan la construcción de políticas. Detrás de cada decisión suele haber una serie de informes, diagnósticos, recopilación de información comparativa, o análisis de alternativas, sin los cuales la intuición o la ocurrencia primarían sobre la valoración detenida de las posibilidades existentes y la posterior toma de decisiones.

Por ese motivo, de manera paralela a la elaboración y tramitación del real decreto mencionado tuvimos que dedicar tiempo y atención a la constitución de los nuevos equipos de trabajo. Como en tantas otras facetas de la actividad política, no existen reglas fijas ni protocolos estrictos para llevar a cabo esta tarea. No sé si afortunada o desgraciadamente, la intuición, el buen sentido, la reflexión y el contraste de opiniones son los criterios que se suelen aplicar, bien es cierto que con diferentes resultados. En nuestro caso, tuvimos un especial cuidado en lograr una combinación de capacidades y competencias personales entre nuestros colaboradores: autoridad y reconocimiento profesional, conocimiento de los distintos niveles educativos, experiencia en la administración educativa, implicación directa en las tareas de enseñanza, interlocución política, sindical o asociativa, capacidad de comunicación oral y escrita, conocimiento internacional, independencia de criterio, entre otras. Además, hubo que cuidar el conjunto, integrando personas procedentes de la administración y de la docencia, de diversas comunidades autónomas, de distintas sensibilidades políticas y sindicales, de distintos cuerpos docentes y administrativos. Sin entrar en detalles, debo señalar que esta tarea exige una atención especial, puesto que resulta clave para un buen desarrollo de la actuación política. Es verdad que en los distintos países existen diferentes tradiciones en lo que se refiere al tamaño de los equipos, a su estabilidad o a su procedencia, por lo que no hay reglas universales. En nuestro caso, aplicamos los criterios habituales en nuestra administración, constituyendo equipos bien compenetrados, pero respetando escrupulosamente los derechos adquiridos y la lógica heterogeneidad de procedencias, lo que constituyó un buen recurso para conseguir análisis amplios y visiones complementarias.

Desde el punto de vista del estudioso de las políticas educativas, creo que se debe subrayar la importancia de prestar la debida atención a la composición de los equipos responsables de su concepción y desarrollo. Y me parece muy recomendable llevar la mirada más allá de lo evidente, esto es, de quiénes son los principales actores y responsables de dichas políticas. 
El estudio de las sinergias y de las contradicciones dentro de los equipos explica algunas fortalezas o debilidades de las políticas adoptadas. El caso de la reforma de la formación inicial del profesorado, en la que confluían visiones no siempre coincidentes entre el mundo universitario y el educativo, es seguramente uno de los que mejor evidencian la cercanía o la distancia que existió en distintos momentos entre los equipos responsables de ambos sectores.

\section{LA IDENTIFICACIÓN DE LA META Y LA ESTRATEGIA A SEGUIR}

Una vez despejadas las urgencias planteadas por la aplicación de la LOCE, comenzamos el proceso que conduciría finalmente a la promulgación de la LOE en 2006. En una primera fase, los esfuerzos estuvieron encaminados a identificar con claridad cuáles eran la meta final que pretendíamos alcanzar y la estrategia más adecuada que debíamos seguir.

Dos eran las opciones que se nos planteaban. La primera consistía en retocar los aspectos más polémicos de la LOCE, para lo que bastaría con promover una ley de alcance limitado, que modificase esta y recuperase algunas de las disposiciones que se habían derogado de la Ley Orgánica reguladora del Derecho a la Educación (LODE) de 1985 y de otras leyes de la etapa de gobierno socialista. La segunda aspiraba a promover una ley general de ordenación educativa, que revisase no solo la LOCE sino también una gran cantidad de artículos que permanecían vigentes de leyes anteriores. Si el recurso a una ley parcial tenía la ventaja indudable de su sencillez, elaborar una ley de carácter general tenía grandes ventajas con vistas a conseguir una mayor coherencia y simplificación de la normativa existente. Los estudios que llevamos rápidamente a cabo de la situación normativa del sistema educativo español pusieron de relieve que la derogación incompleta que se había hecho con el paso de los años de la Ley General de Educación (LGE) de 1970, la Ley Orgánica de Ordenación General del Sistema Educativo (LOGSE) de 1990, la Ley Orgánica de Participación, Evaluación y Gobierno de los Centros Docentes (LOPEG) de 1995, así como de todos los decretos y órdenes dictados para sus desarrollos respectivos, complicaba considerablemente el panorama normativo aplicable a la educación. Por lo tanto, derogarlas todas ellas sería un objetivo adicional, lo que implicaba integrar sus disposiciones residuales en la nueva ley o eliminarlas, según los casos. 
La voluntad de racionalidad normativa nos aconsejaba elaborar una ley general, que reuniese la legislación dispersa, la simplificase y la adaptase al nuevo marco competencial establecido por la Constitución de 1978 y los Estatutos de Autonomía. Y desde el punto de vista de la ordenación educativa, ofrecía además la ventaja de reforzar la coherencia del sistema. Sin duda, era la opción que preferíamos. Pero a esas ventajas, a nuestro juicio poderosas, se oponía el serio inconveniente de la dificultad política que dicha opción entrañaba. Al no contar el Gobierno con una mayoría absoluta en el Congreso ni en el Senado, estaríamos obligados a buscar acuerdos parlamentarios, a conseguir que otros grupos políticos se sumasen al proyecto y le diesen su voto favorable. Salvo en el caso de la LOPEG, una ley de menor alcance que la ahora propuesta, todas las leyes antes mencionadas habían sido aprobadas por gobiernos con mayorías parlamentarias absolutas. Aunque eso no les había impedido sumar apoyos importantes (como fue por ejemplo el caso de la LOGSE), el acuerdo parlamentario no había sido un requisito ineludible, como sucedía ahora. Además, al haberse pospuesto la aplicación de la LOCE hasta 2007, ese era el plazo límite para aprobar una nueva ley que sustituyese a la anterior, so riesgo de tirar por la borda todo el esfuerzo realizado. Esa circunstancia fue objeto de un análisis cuidadoso, tanto en el ministerio como en la Presidencia del Gobierno. Nuestra propuesta de elaborar una ley general de educación fue sometida a un escrutinio riguroso, contrastada con la correlación de fuerzas existente en el Parlamento, valorada desde el punto de vista técnico y político y finalmente adoptada. El proceso fue intenso y exigente, pero al final del mismo quedó clara la meta a lograr. Teníamos un plazo de dos años para elaborar, negociar y aprobar una ley general de educación.

Viviendo este proceso desde el interior, resulta muy interesante comprobar cómo se desarrolla cada uno de los pasos que llevan a identificar la meta a conseguir. Especialmente valioso resulta comprobar el peso de los factores no estrictamente educativos, sobre todo los de naturaleza política, en la toma de decisiones. No puede decirse que los elementos educativos de la decisión no se tomasen en consideración, pero puede sorprender el análisis desapasionado que se llevó a cabo de las circunstancias políticas concretas, que podían condicionar muy notablemente el resultado. Y por otra parte, también llama la atención la importancia que tiene la existencia de un grupo impulsor cohesionado y bien preparado, como era el caso, a la hora de vencer determinadas resistencias internas que se plantean por desconocimiento o desconfianza en el proyecto. 
Una vez identificada la meta, hay que prestar atención al diseño de una estrategia que permita alcanzarla. En el caso de la LOE, el propio programa electoral del PSOE de 2004 y la convicción del equipo ministerial apuntaban claramente a que la clave consistía en crear complicidades para sumar apoyos al proyecto. Para ello, lo primero debía ser propiciar un proceso de diálogo, cuya carencia se había destacado como un punto negativo en la elaboración de la LOCE. Así, el programa electoral decía expresamente:

Basándonos en la colaboración mutua, la lealtad institucional y la corresponsabilidad, propiciaremos un diálogo con todos, en primer lugar con las Comunidades Autónomas, con Ayuntamientos, Comunidad Educativa, CRUE, agentes sociales, organizaciones educativas y escolares, científicas y de comunicación para lograr un consenso básico sobre los cambios necesarios para lograr los objetivos comunes. ${ }^{6}$

Esas palabras trazaban el camino a seguir, aunque todavía estábamos obligados a diseñar el proceso concreto que deberíamos desarrollar. Y esa convicción en el poder del diálogo presidió nuestros esfuerzos de la etapa siguiente.

\section{EL DIALOGO COMO INSTRUMENTO DE CONSTRUCCIÓN DE POLÍTICAS}

Con el propósito de llevar a cabo la estrategia así definida, decidimos abrir un proceso de debate con carácter previo a la elaboración del proyecto de la nueva ley. Conviene recordar que esta práctica política contaba con una larga tradición en España, iniciada más de treinta años antes, en un contexto muy diferente, con la publicación del Libro Blanco previo a la Ley General de Educación. ${ }^{7}$ Acerca de aquella iniciativa Manuel de Puelles ha afirmado que "la verdad es que nunca en la Administración española se había abordado una reforma desde presupuestos técnicos tan rigurosos» ${ }^{8}$ Tras esa primera experiencia, se llevaron a cabo otros procesos similares de

\footnotetext{
6 PSOE, Merecemos una España mejor, 167.

7 MEC, La educación en España. Bases para una política educativa (Madrid: Ministerio de Educación y Ciencia, Secretaría General Técnica, 1969).

${ }^{8}$ Manuel de Puelles, «Tecnocracia y política en la reforma educativa de 1970», Revista de Educación, n. ${ }^{\circ}$ extraordinario (1992): 19.
} 
debate en el proceso de elaboración de la LOGSE y de la LOPEG, aunque no fuese el caso de la LOCE. Queríamos que la elaboración y discusión de la que finalmente sería la LOE continuase esa tradición, que considerábamos profundamente democrática y participativa y que he tenido ocasión de analizar con mayor detalle en otro lugar. ${ }^{9}$

Para propiciar un debate constructivo y fructífero, comenzamos por elaborar un documento que permitiese centrar la discusión en los puntos a nuestro juicio críticos de la ordenación del sistema educativo español y que requerían una transformación urgente. Es así como surgió la publicación Una educación de calidad para todos y entre todos. Propuestas para el debate, que fue ampliamente distribuida y también incluida en la página web debateeducativo.mec.es, creada específicamente con el objetivo de sustentar el posterior proceso de debate. ${ }^{10} \mathrm{El}$ documento no pretendía ser un informe académico, sino una guía para la reflexión y la consulta previas a la toma de decisiones. Con ese propósito, se organizaba en siete bloques, con un total de catorce capítulos, en los que se abordaban asuntos tales como las características de las diversas etapas educativas, las competencias y saberes necesarios para los ciudadanos del siglo XXI, los valores que deben cultivarse en la escuela y el sentido de la formación ciudadana, el desarrollo de la profesión docente, la elección de centro y su pluralidad, y la organización y autonomía de los centros docentes. Cada uno de los capítulos incluía un diagnóstico de la situación, continuaba con un conjunto de propuestas concretas y finalizaba con una serie de preguntas pensadas para promover el debate.

Vale la pena destacar que, como se ha indicado, no se trataba de una estrategia estrictamente novedosa, puesto que había sido aplicada en varias ocasiones desde el año 1969. Pero sí que respondía a una voluntad de búsqueda de complicidades y de acuerdos que permitiesen elaborar y aprobar una ley con garantías de aceptación y con los apoyos suficientes. A fin de cuentas, las circunstancias políticas obligaban a ello. Y por otra parte, queríamos marcar claramente las diferencias con el proceso de ela-

\footnotetext{
${ }^{9}$ Alejandro Tiana, «El recurso al debate público en los procesos de reforma educativa: Análisis de la experiencia española (1969-2012)», Multidisciplinary Journal of Educational Research, 3 (1), (2013): 19-41 (consultado el 30 de mayo de 2015), doi: 10.4471 /remie.201 3.02.

${ }^{10}$ MEC, Una educación de calidad para todos y entre todos. Propuestas para el debate, (Madrid: Ministerio de Educación y Ciencia, 2004). Continúa estando accesible (a 30 de mayo de 2015) en http://debateeducativo.mec.es/pdf/libro_educacion.pdf
} 
boración de la LOCE, que había despreciado el diálogo como instrumento de construcción de la política educativa. Eso había dicho con claridad meridiana la ministra Pilar del Castillo durante el proceso de aprobación de la LOCE:

Las cosas hay que hacerlas sin esperar el sí de todos, sería imposible y, además, una falta de responsabilidad. Si un político lo que espera es tener la palmada en el hombro de la oposición o no sabe de lo que está tratando o no está haciendo nada [...] el tiempo de debate ha de ser el suficiente para hacer las cosas con rigor pero el tiempo no es el objetivo fundamental de una reforma, el objetivo es hacerla (...) El diálogo no se puede confundir con una tertulia de café o de amigos que no tiene más objetivos que hablar y hablar un día y otro día. El diálogo tiene una finalidad, si esa finalidad no se comparte se fracasa. Si el objetivo es hacer una reforma del sistema educativo y hay quien no tiene esa intención y lo que quiere es poner pequeños parches o hacer las cosas en una dirección diametralmente opuesta a la de uno, el diálogo se inicia pero se frustra. ${ }^{11}$

Ante declaraciones de este tenor, la estrategia política basada en el diálogo pretendía poner en evidencia a quienes habían prescindido del mismo en la elaboración de la LOCE. Se quería demostrar que era posible construir políticas de un modo diferente. Creíamos además en ese momento que la insistencia en este tipo de prácticas marcaría una dirección que resultaría difícilmente reversible aunque, a la vista de la frustrante experiencia de debate previo a la LOMCE, está claro que nos equivocamos. Esto me hace pensar nuevamente en la fuerza tan considerable que tienen las circunstancias políticas y las tradiciones partidarias en los procesos de construcción de políticas de la educación, más allá del peso que solemos atribuir al peso de la racionalidad.

El proceso de debate ha sido ampliamente expuesto en otro lugar, por lo que no me extenderé en esta ocasión en su presentación. ${ }^{12}$ Tan solo recordaré que el documento Una educación de calidad para todos y entre todos fue pre-

\footnotetext{
${ }_{11}$ Entrevista a Pilar del Castillo, Boletín del Ilustre Colegio de Doctores y Licenciados en Filosofía y Letras y en Ciencias, 137 (septiembre 2002): 3-7.

${ }_{12}$ Alejandro Tiana, "A la búsqueda del consenso en educación: la experiencia de la LOE», Revista de Educación, 344 (2007): 83-100.
} 
sentado formalmente por la ministra de Educación y Ciencia, María Jesús San Segundo, en el mismo día a la Conferencia sectorial de Educación y al Consejo Escolar del Estado, lo que implicaba hacerlo público a los gobiernos autonómicos, por una parte, y a las organizaciones educativas y sociales, por otra. Ese acto representó la apertura del proceso de debate, que se desarrolló en un triple plano: con las comunidades autónomas, fundamentalmente a través de la Comisión General de la Conferencia sectorial de Educación, en la que participan los viceconsejeros autonómicos; con los representantes sociales, a través del Consejo Escolar del Estado y sus comisiones y de los Consejos Escolares autonómicos; y con la comunidad educativa, a través de reuniones, actos públicos, emisión de informes y participación en el portal abierto al efecto. Los números dan una idea de la amplitud del debate. En los seis meses que duró el proceso se recibieron 304 documentos de organizaciones, asociaciones y grupos diversos, que daban respuesta a las cuestiones planteadas y que fueron hechos públicos, de modo que cualquier persona pudiese consultar las opiniones manifestadas por unos y otros, ${ }^{13}$ llegaron otros 46.000 documentos más breves, en forma de cartas, faxes o mensajes de correo electrónico, se registraron más de 21.000 aportaciones en los seis foros abiertos en Internet y se celebró un elevado número de actos públicos.

Reflexionando nuevamente sobre las características de este proceso de debate, orientado a alcanzar algunos acuerdos, me reafirmo en dos observaciones relativas al proceso seguido que me parecen claves y ya he tenido ocasión de analizar. ${ }^{14}$ La primera tiene que ver con el grado de reserva de los debates mantenidos o, dicho a la inversa, con el grado de exposición mediática de las conversaciones. Así, mientras que el debate mantenido en la Comisión General de Educación tuvo un carácter reservado, el registrado en el Consejo Escolar del Estado adquirió una amplia repercusión mediática. Como he tenido ocasión de explicar, ello se debe a que, después de cada reunión, algunos consejeros acudían a los medios de comunicación, con la intención de hacer públicas sus posturas y contraponerlas a las de sus opositores. En consecuencia, la discusión de las propuestas se planteó en los órganos del Consejo y de manera paralela en los medios, con el efecto añadido de que algunos temas encontraron una gran resonancia mediática, a

\footnotetext{
${ }^{13}$ Dicho sea de paso, exactamente lo contrario ocurrió con el falso debate previo a la aprobación de la LOMCE, donde resultó imposible conocer las contestaciones de las personas y organizaciones que respondieron a las propuestas del Ministerio de Educación, Cultura y Deporte.

${ }^{14}$ Alejandro Tiana, «A la búsqueda del consenso en educación».
} 
costa de otros que pasaron más desapercibidos. La segunda diferencia tiene que ver con el proceso de adopción de acuerdos. Mientras que el Consejo recurrió sistemáticamente a las votaciones, pese a que no se trataba de aprobar un dictamen preceptivo, la Comisión General de Educación no resolvió ningún asunto por votación, limitándose a tomar nota de los acuerdos alcanzados y las discrepancias manifestadas, sin pretender formular una propuesta mayoritaria cuando había división de opiniones. En consecuencia, en el Consejo se produjeron mayores enfrentamientos ideológicos que en la Comisión General de Educación, además de que fuesen amplificados por los medios de comunicación. El resultado fue que en el Consejo se formaron bandos de ganadores y perdedores, mientras que en la Comisión General se pudieron buscar puntos de acuerdo de manera más libre y dedicando todo el tiempo necesario a avanzar en cada asunto, sin presiones externas.

Entiéndase bien que no estoy argumentando con esto a favor de la falta de transparencia, sino de la necesidad de asegurar las condiciones de un debate libre, en el que se puedan explorar los límites de las posiciones respectivas y por tanto de los posibles acercamientos en aras de un acuerdo. No creo que deba interpretarse la exposición a los medios como un procedimiento más democrático y abierto, sino como uno que introduce criterios externos al propio proceso de negociación, como son los usos de la comunicación pública, los intereses e ideología de los propios medios, el diferente grado de preparación de los comunicadores y los juegos de influencia que se dirimen en esa transmisión. En suma, quiero insistir en que no estamos solo ante una cuestión de apertura y transparencia en el debate, sino de cuidar de que no se tergiversen las reglas de cualquier negociación que pretende alcanzar acuerdos y de evitar quedarse solamente en enfatizar las diferencias para vencer al contrario.

Una demostración adicional del impacto negativo de la publicidad mediática de los procesos de negociación tiene que ver con el intento que se produjo de lograr un acuerdo social en torno al proyecto de ley, promovido por la Federación de Enseñanza de Comisiones Obreras (CCOO), la Federación de Trabajadores de la Enseñanza (FETE-UGT), la Federación Española de Religiosos de la Enseñanza (FERE-CECA), la Confederación Española de Asociaciones de Madres y Padres de Alumnos (CEAPA) y la Confederación Católica Nacional de Padres de Familia y Padres de Alumnos (CONCAPA), y en el que actuaron como mediadores, a petición de sus promotores, Manuel de Puelles y Agustín Dosil, miembros ambos del Consejo Escolar del Estado, 
con posiciones ideológicas diferentes y una merecida reputación de personas dialogantes. Con el propósito de alcanzar un acuerdo básico, al que luego se sumaran otras organizaciones, mantuvieron reuniones de carácter reservado en las que fueron discutiendo y acordando un texto conjunto, bien es cierto que no sin dificultades. Pero, cuando parecía que las condiciones eran favorables para firmar el documento que plasmaba el pacto logrado y sólo faltaba la decisión formal de alguna de las organizaciones implicadas, el proceso cambió súbitamente de rumbo. Tal intención trascendió a los medios, seguramente por alguna filtración interesada, y se generó una controversia pública que influyó directamente en las organizaciones participantes. En algunas de ellas aparecieron tensiones internas entre los partidarios y los detractores del pacto, otras sufrieron fuertes presiones externas para no firmarlo y, en general, el ambiente se hizo menos favorable para alcanzar acuerdos. Todo apunta a que hubo sectores, tanto internos como externos a dichas organizaciones, muy interesados en que no se alcanzase ningún tipo de acuerdo, que lograron influir en el resultado final. Creo que la experiencia es muy reveladora y vale la pena reflexionar sobre ella.

Antes de pasar a otro asunto, debo señalar que uno de los puntos débiles del debate mantenido consistió en la limitada participación en el mismo del conjunto de la comunidad educativa. Si bien, como he señalado, sus representantes y las principales organizaciones participaron activamente en el debate, e incluso promovieron intentos de pacto y acuerdo bien orientados, la participación de los docentes, estudiantes, familias, centros educativos y otros agentes fue mucho menor. He hablado más arriba de varios miles de comunicaciones, entradas en la web y envío de documentos, pero las cifras no son muy elevadas en comparación con las dimensiones del sistema educativo. Claude Thélot, conductor de una importante experiencia de debate sobre el futuro de la escuela llevada a cabo en Francia entre 2003 y 2004, señalaba la importancia de recoger la opinión de las personas alejadas de dicho debate, llegando a considerarla una precaución ineludible. ${ }^{15}$ Es cierto que hubo un cierto número de centros docentes que participaron en el debate previo a la LOE, pero en número muy limitado. Cuando se habla de la necesidad de implicar al profesorado y a la comunidad escolar en los procesos de cambio educativo, esta falta de participación resulta especialmente grave. Si hasta los años noventa resultaba ciertamente complicado

${ }^{15}$ Claude Thélot, Débattre pour réformer. L'exemple de l'École (Paris: Dunod, 2005). 
lograr que los centros educativos dejasen oír su voz, la reciente expansión del uso de las tecnologías de la información y la comunicación la ha facilitado mucho. Y sin embargo, esa generalización del uso de los nuevos canales de comunicación no ha incrementado la participación tanto como hubiese sido deseable.

En cualquier caso, creo que puedo concluir este apartado repitiendo lo que decía en el artículo mencionado más arriba:

En conjunto, puede afirmarse que el proceso de debate fue amplio, rico y variado. El Ministerio de Educación y Ciencia tuvo la ocasión de cotejar sus propuestas con los principales actores del mundo educativo, y, de este modo, pudo detectar en qué aspectos existía la posibilidad de alcanzar acuerdos y en cuáles sería una tarea más difícil. Para los responsables del MEC, los objetivos del debate se cumplieron muy satisfactoriamente. ${ }^{16}$

\section{EL PROCESO INICIAL DE NEGOCIACIÓN SOCIAL Y POLÍTICA}

El periodo de debate sirvió para ir elaborando el primer borrador de lo que acabaría siendo la nueva ley de educación. Aunque ya disponíamos de un esquema preliminar antes de finalizar 2004, la conversión de ese bosquejo inicial en un proyecto articulado se llevaría a cabo durante los primeros meses del año 2005. De acuerdo con tal cronología, resulta fácil entender que el proceso de debate público permitió contrastar las propuestas iniciales y aportó sugerencias valiosas para preparar el texto legal. Además, de manera paralela, tuvo lugar un primer proceso de negociación, en un doble plano, social y político, al que merece la pena referirse expresamente.

Antes de entrar en ese análisis, conviene señalar que la elaboración del borrador de la ley se hizo con sumo cuidado. Se analizaron con detalle las partes que debería incluir, sus elementos fundamentales, su organización y su orden de prelación, el tipo de disposiciones que tendrían que formularse y la distinción entre artículos básicos y no básicos. Las articulaciones internas de la ley y las conexiones con otras normas legales fueron objeto de especial atención, de igual modo que se cuidó el rigor jurídico del texto resultante. Pero aunque recibimos propuestas de muchas procedencias, man-

${ }^{16}$ Alejandro Tiana, «A la búsqueda del consenso en educación», 92. 
tuvimos múltiples conversaciones, solicitamos aportaciones sobre diversos aspectos concretos y consultamos reiteradamente con nuestros asesores jurídicos, la preparación del texto legal fue tarea de un grupo reducido de componentes del equipo de la Secretaría General de Educación. La composición plural de dicho grupo, formado por personas con formación pedagógica, administrativa y jurídica, resultó fundamental para conseguir un buen resultado. Y el hecho de que el trabajo fuese responsabilidad de un grupo de esas características permitió elaborar un texto coherente, bien trabado y con sentido de unidad. En todo caso, no se debe ignorar que sobre la mesa en que mantuvimos sesiones de trabajo casi diarias teníamos informes diversos, aportaciones parciales sobre los diferentes títulos o capítulos de la ley e informes de especialistas. Todo el amplio equipo de nuestros colaboradores más cercanos se pudo sentir partícipe de un proceso en el que todos tuvieron ocasión de participar en una u otra forma.

Quiero destacar que esa combinación de un trabajo intenso en un grupo de dimensiones limitadas y una decidida apertura a una multiplicidad de aportaciones externas permitió llevar a cabo una experiencia rica, que redundó en lo que sigo creyendo que fue un buen resultado. Me atrevo incluso a decir que se trata de un buen procedimiento de trabajo para la formulación de políticas sectoriales, en este caso en el campo de la educación, por cuanto permite combinar una perspectiva amplia y poliédrica, necesaria para abarcar un ámbito completo, con la ineludible articulación y coherencia de la norma resultante. No he tenido otras experiencias similares, pero reconozco que esta marcó la pauta para los proyectos que continuamos desarrollando en los años inmediatos y me ha servido incluso de guía en etapas profesionales posteriores.

La elaboración del texto no estuvo exenta de dificultades, al tratarse de una ley general de educación. En efecto, muchos de los artículos que aún quedaban vigentes de la LOGSE, la LOPEG o la propia LOCE debían ser reformulados e integrados en la nueva ley. Ello tenía varias implicaciones, entre las que quiero destacar especialmente dos: por una parte, la formulación que se adoptase debía acomodarse al carácter de la ley y a los criterios que el Tribunal Constitucional había ido desarrollando y exigiendo para las piezas de legislación básica; por otra parte, aunque algunos preceptos de aquellas leyes estuviesen aún vigentes, su inclusión en la ley que pergeñábamos obligaba a discutirlos y aprobarlos de nuevo en su trámite parlamentario. El camino elegido planteaba dificultades de este tipo, que no podían soslayarse. 
En estas circunstancias, resultaba ineludible emprender una negociación a varias bandas con carácter previo a la aprobación del proyecto de ley por parte del Gobierno. Ante todo, resultaba necesario asegurar que el texto que se preparaba pudiese contar con la aceptación de las organizaciones representativas del mundo educativo, tales como los sindicatos del profesorado, las asociaciones de padres y madres, las organizaciones de estudiantes y las organizaciones de titulares de la enseñanza privada y concertada (o al menos evitar su oposición). Con ese propósito, dentro del proceso de debate se llevaron a cabo múltiples reuniones con tales entidades, de carácter bilateral unas veces y colectivo otras. Con todas ellas mantuvimos conversaciones, exploramos su posición respecto de los aspectos más debatidos del proyecto, recibimos sus comentarios, reacciones y sugerencias, y negociamos algunas formulaciones concretas. Debo decir que la tarea no resultó sencilla, pues en ocasiones las posiciones estuvieron abiertamente enfrentadas, buscando un pronunciamiento ministerial a favor o en contra de determinadas propuestas, que podía en el extremo invalidar la búsqueda de acuerdos. En algunos casos, las negociaciones se inscribieron en marcos más amplios que los referidos estrictamente a esta ley, como fue el caso de algunas posiciones de la Federación Española de Religiosos de Enseñanza (FERE), condicionadas por la posición de la Conferencia Episcopal en contra de políticas concretas del gobierno, como pudo ser el caso de la ley relativa al matrimonio homosexual y su traducción en la Educación para la Ciudadanía y los Derechos Humanos. Cabe mencionar que el hecho de que en ese mismo periodo de tiempo estuviese desarrollándose el intento de pacto educativo antes mencionado, que contó con la participación activa de algunas de tales organizaciones, facilitó la búsqueda de acuerdos. Y asimismo vale la pena señalar que, si bien dicho pacto se demostró finalmente inalcanzable, el documento suscrito por las organizaciones negociadoras sirvió de base para el desarrollo de algunos apartados concretos de la ley. Aunque el pacto no se hubiese logrado firmar, la voluntad de acuerdo y las formulaciones alcanzadas resultaron muy valiosas para iluminar el espacio de consenso y fueron en consecuencia tenidas en cuenta por el equipo redactor e incluso algunas de ellas incorporadas a la nueva ley.

Aunque lo haya puesto de relieve en otros lugares y circunstancias, no quiero dejar de recordar aquí que la ministra de Educación y Ciencia, María Jesús San Segundo, había dejado explícita su voluntad de búsqueda de 
acuerdos en la introducción que hizo al documento Una educación de calidad para todos y entre todos:

si se alcanza un amplio acuerdo social, por el que trabajaremos sin descanso, los responsables educativos podrán aportar sus criterios y sus principios, una vez garantizada una base común estable que cuente con el mayor apoyo social posible. Este es el objetivo principal del debate que ahora iniciamos, y del proyecto de Ley que llevaremos al Parlamento. Sólo se puede lograr una educación de calidad para todos los jóvenes españoles si el conjunto de la sociedad participa en la definición del sistema educativo y lo apoya en su desarrollo. ${ }^{17}$

En consecuencia, la voluntad de acuerdo no era una simple estrategia, por inteligente que pudiese resultar en el ambiente de controversia y conflicto en que se había aprobado la LOCE, sino fruto de una inequívoca decisión política, basada en la convicción de que solamente el consenso permitiría lograr la estabilidad tan reclamada —entonces y aún en la actualidad- por muy diversos sectores del mundo educativo.

Una muestra simbólica de la voluntad de consenso y búsqueda de acuerdos que presidió la elaboración del texto legal se encuentra en su denominación. En efecto, a la hora de revertir la LOCE, que había hecho de la calidad de la educación su bandera, hubo quien defendió que enarbolásemos otra, la de la equidad, concebida como el principio central de la nueva ley. Sin embargo, decidimos huir de ese tipo de enfrentamientos y preferimos adoptar un nombre simple y descriptivo, que pudiese ser aceptado por cualquiera y no generase rechazos o tomas de posición contrarias. Es así como surgió la propuesta de denominación de Ley Orgánica de Educación, que fue la finalmente asumida.

Siendo importante, la negociación con las organizaciones del mundo educativo no era suficiente. Ante la necesidad de contar con aliados parlamentarios para aprobar la ley en el Congreso y el Senado, decidimos negociar el borrador que íbamos construyendo con los dos grupos políticos que se mostraron más proclives al acuerdo. Se trataba de Esquerra Republicana de Catalunya (ERC) e Izquierda Unida - Iniciativa per Catalunya Verds

${ }^{17}$ MEC, Una educación de calidad para todos y entre todos, 5-6. 
(IU-ICV). El hecho de que ambos formasen parte, junto con el Partit dels Socialistes de Catalunya (PSC), del gobierno tripartito de Cataluña facilitó además el acercamiento. Por otra parte, la consejera de Educación pertenecía a ERC, motivo por el cual manteníamos contactos muy frecuentes. Así pues, el borrador que se fue elaborando en los primeros meses de 2005 fue discutiéndose paralelamente con ambos grupos políticos con representación parlamentaria.

Si bien había quien vaticinaba la dificultad de llegar a acuerdos concretos sobre la ley con estos dos grupos, especialmente en lo que se refiere a la distribución de competencias en materia de educación entre la Administración central y las comunidades autónomas, asunto complejo y no exento de tensiones, ${ }^{18}$ la experiencia fue otra. Formamos un grupo estable de trabajo, que fue discutiendo de manera sistemática todos los aspectos de la ley y buscando acuerdos sobre todos ellos. Las discusiones fueron intensas, las negociaciones en ocasiones difíciles, especialmente con IU-ICV por la diversidad interna y territorial de sus componentes, pero la voluntad de acuerdo prevaleció sobre las discrepancias. Conviene señalar que la coincidencia en la idea de que la LOCE era una ley rechazable y que urgía revertirla facilitó el acuerdo, incluso en algunos puntos en que existían divergencias acerca de cuál fuese la mejor solución para reemplazarla. Es así como elaboramos finalmente un borrador aceptado por ERC e IU-ICV, además de contar con el beneplácito de las consejerías de Educación de las comunidades autónomas con gobierno socialista, con las que mantuvimos un contacto permanente.

Como más adelante tendré ocasión de analizar detenidamente, ese modo de proceder tuvo muchas ventajas, pero también presentó algunos inconvenientes. Entre las primeras hay que subrayar sobre todo la tranquilidad de saber que el proyecto de ley contaría con un amplio acuerdo en su trámite parlamentario, asunto que preocupaba extraordinariamente al propio ministerio y a la Presidencia del Gobierno. Aunque siempre se pudiesen volver a discutir y negociar algunos flecos determinados, teníamos la convicción de que la aprobación de la ley era factible. Entre los inconvenientes, quiero mencionar uno del que no tuvimos conciencia en un primer momento, pero que acabó teniendo cierta importancia. Se trata del hecho de que el texto

\footnotetext{
${ }^{18}$ Sobre este asunto puede consultarse Alejandro Tiana, «La coordinación sectorial en materia de educación en España», Revista de Administración Sanitaria, 3 (5), (2007): 373-389.
} 
de la ley llegaba al Congreso muy acordado con ERC e IU-ICV, por lo que quedaba poco espacio para realizar modificaciones en el recorrido parlamentario.

Me explicaré con mayor detalle, porque esta es una reflexión relevante. En efecto, los puntos más debatidos de la ley habían sido objeto de negociación y de acuerdo previo, incluyendo en el texto la redacción de cada uno de ellos que resultaba más adecuada y admisible para todas las partes negociadoras. En algún momento sugerimos dejar pendiente algún aspecto ya acordado para introducirlo posteriormente en el debate parlamentario. Aunque debo reconocer que la negociación fue franca, no dejaba de subyacer una cierta desconfianza, sobre todo por parte de ERC e IU-ICV hacia el PSOE, de manera tal que prefirieron llegar al debate parlamentario con el texto ya cerrado. En consecuencia, el proyecto de ley que aprobó el Gobierno en julio de 2005 y remitió al Congreso para su tramitación les dejaba tranquilos, puesto que lo asumían como propio y constituía una clara demostración de la voluntad de acuerdo. Y también la publicación de una memoria económica de unos siete mil millones de euros para la aplicación de la LOE durante cinco años, la mayor de cualquiera de las leyes educativas aprobadas en España hasta el momento en que escribo estas líneas, fue resultado de las presiones de ambos grupos. En suma, estamos hablando de un grado muy importante de acuerdo, concretado en elementos tangibles. Pero, al mismo tiempo, la estrategia de acuerdos previos dejaba poco espacio para escenificar durante el trámite parlamentario el acercamiento que había tenido lugar.

Debo añadir que el hecho de que el texto estuviese acordado de manera bastante detallada no excluía la posibilidad de modificarlo durante el trámite parlamentario, como resultado de las negociaciones que pudiesen mantenerse con otros grupos políticos, si bien dichas modificaciones deberían ser acordadas por los tres grupos promotores. Aunque hubo muchas personas y grupos que creyeron imposible que tal tipo de concesiones pudiesen ser aceptadas, la realidad fue otra, como mencionaré en el apartado siguiente.

Es sabido y se ha mencionado muchas veces que la política tiene algo (o mucho) de representación escénica y el trabajo parlamentario es quizás la quintaesencia de dicha escenificación. Creo que todas las personas que hemos tenido esa experiencia coincidimos en dicha apreciación, que debe ser 
tenida en cuenta cuando analizamos la práctica política y sus resultados. No quiere ello decir, entiéndase bien, que sean actos falsos, meras actuaciones, pues el fondo de los asuntos que se tratan suele ser muy relevante y los acuerdos y discrepancias son reales y claramente identificables. Me refiero a que el modo de presentar los acuerdos y las desavenencias está orientado no solo a lograr un buen resultado en las votaciones que puedan producirse, sino también a su recepción e interpretación favorable por un público externo. De ahí deriva, en mi opinión, el carácter de representación al que antes aludía.

En este caso, la tramitación parlamentaria permitió llegar a acuerdos con otros grupos políticos, algunos de los cuales mostraron como logros importantes los cambios introducidos, independientemente de su calado. Y así, por ejemplo, mientras que CiU subrayó con trazo bien visible las modificaciones que se introdujeron en la ley como efecto de su capacidad negociadora, ERC e IU-ICV no pudieron mostrar del mismo modo su destacada incidencia en el texto, sin duda mucho mayor y más relevante, al venir este ya cerrado en el borrador aprobado por el Gobierno. Y este hecho no dejó de producir algunas turbulencias en la fase de aprobación parlamentaria de la LOE.

\section{LA NEGOCIACIÓN PARLAMENTARIA}

Aunque el proyecto de ley llegó al Congreso con garantías razonables de aprobación, como ya se ha explicado, la voluntad de consenso expresamente manifestada llevó a intentar sumar a otros grupos políticos a ese acuerdo inicial. En esta fase de discusión parlamentaria, la dirección del proceso correspondió al grupo parlamentario socialista, ${ }^{19}$ si bien el equipo ministerial continuó manteniendo una participación muy activa.

Las negociaciones se produjeron en esta fase con todos los grupos con representación parlamentaria. Las más complicadas fueron sin duda las mantenidas con el Partido Popular (PP). A lo largo de varias sesiones de trabajo analizamos conjuntamente el proyecto y tuvimos ocasión de debatir acerca de las notorias discrepancias existentes. Algunas de ellas ya habían

\footnotetext{
${ }^{19}$ Hay que recordar que el entonces portavoz del Grupo Parlamentario Socialista, Alfredo Pérez Rubalcaba, se implicó decididamente en el proceso, lo que aportó notable fuerza política a las negociaciones mantenidas. Es necesario mencionar también el papel destacado que desempeñó en ese proceso Raimundo Benzal, ponente socialista de la ley.
} 
sido puestas de relieve por las consejerías gobernadas por el PP, en el seno de la Comisión General de Educación, aunque ahora la discusión tuvo un carácter más político. Hay que reconocer que las conversaciones permitieron encontrar algunos puntos de acuerdo, como los relativos al porcentaje de especificación del currículo por parte de la Administración central, el carácter propedéutico del $4 .^{\circ}$ curso de la Educación Secundaria Obligatoria (ESO) o la posibilidad de adelantar la participación en los Programas de Cualificación Profesional Inicial (PCPI) a los 15 años de edad en determinados supuestos. No obstante, al producirse en ese mismo periodo la toma en consideración por el Congreso de la reforma del Estatuto de Cataluña no fue posible continuar con las negociaciones. El enfrentamiento político fue de tal calibre que las conversaciones sobre la LOE quedaron suspendidas y el PP presentó una enmienda a la totalidad del proyecto de ley, consistente en ir enmendando cada artículo por medio de un artículo de la LOCE, lo que impedía cualquier negociación con sentido. Vale la pena señalar que hubiese resultado factible encontrar algunos puntos de confluencia entre PSOE y PP, que no violentarían además los acuerdos establecidos con ERC e IU-ICV, pero que la situación política lo hizo imposible. Dicho sea de paso, es algo parecido a lo que sucedió con el proceso de pacto promovido unos años después por el ministro Ángel Gabilondo, que también fue impedido por los intereses políticos del PP, más que por diferencias insalvables acerca de los temas abordados.

También complicada, pero con bastante más voluntad de acuerdo, fue la negociación mantenida con Convergència i Unió (CiU). En este caso había varias discrepancias acerca del reparto competencial establecido, el lugar y la regulación de la enseñanza concertada y otros asuntos menores. El debate fue intenso, pero abierto, y fue posible encontrar algunos puntos de acuerdo que quedaron recogidos en la ley. Como se ha explicado, esos acuerdos fueron la causa de algunas tensiones con ERC, aunque la incidencia de CiU en la LOE fuese mucho menor que la suya.

Merece la pena mencionar aquí también el alto grado de acuerdo encontrado con Coalición Canaria, que además había adoptado un papel mediador en los debates con los representantes autonómicos, la falta de discrepancias graves con el Partido Nacionalista Vasco (PNV), excepto en lo relativo a su rechazo a la competencia municipal en materia de educación, la falta de conflictos graves con otros partidos regionalistas y las entretenidas conversaciones con José Antonio Labordeta, de la Chunta Aragonesista. 
Mantuvimos reuniones con todos los grupos parlamentarios y con los integrantes del grupo mixto, aunque algunas fueron más minuciosas que otras.

Es interesante destacar aquí la relevancia que llegaron a tener algunos asuntos generalmente aceptados como menores, pero que causaron algunas de las mayores dificultades al final de la tramitación parlamentaria de la LOE. Es llamativo que no fuesen los temas con mayor resonancia mediática, como el de la Educación para la Ciudadanía o la enseñanza de la religión católica en la escuela, los que generasen discrepancias graves. No debe entenderse que hubiese acuerdo al respecto, pero el grado de conflicto fue muy reducido. En efecto, sería después de la aprobación de la LOE cuando la polémica se centrase en esas cuestiones, fruto entre otras cosas de unas campañas bien orquestadas de desobediencia civil y de desprestigio de la ley. ${ }^{20}$ Uno de esos asuntos menores que generó discusiones intensas por su carácter simbólico fue la propuesta de incorporación de un representante municipal en los consejos escolares de los centros concertados. Para la FERE se trataba de una cuestión inaceptable, posición que compartía CiU, mientras que para IU-ICV su inclusión estaba fuera de discusión. En el trámite parlamentario en el Senado se eliminó ese apartado, lo que conllevó el voto favorable de CiU y la abstención de IU-ICV, pero en el regreso al Congreso se incorporó de nuevo, lo que tuvo consecuencias opuestas. Como puede verse, aunque no se trataba de un asunto importante, la visualización de la victoria en este punto se vivió como una controversia relevante. El carácter simbólico del asunto pesó más que el cambio que introducía, claramente secundario.

Es interesante señalar al respecto que este tipo de desavenencias pueden llegar a provocar el riesgo de romper un proceso negociador de mucho más calado. Es una experiencia que he tenido ocasión de vivir en distintos contextos y situaciones. Y es que resulta más sencillo llevar adelante un proceso de negociación cuando se ofrecen varias opciones para resolver diversos puntos de discrepancia, con lo que se pueden realizar cesiones en algunos de ellos

\footnotetext{
${ }^{20}$ Véase al respecto mis trabajos: «La Enseñanza Religiosa Escolar: los planteamientos del Ministerio de Educación y Ciencia», Bordón, 58 (4-5), (2006): 569-582; «Educación para la Ciudadanía y los Derechos Humanos: el sentido de una novedad», Temas para el debate, 149 (2007): 42-44; «Educación para la ciudadanía: una nueva propuesta para la formación cívica de los jóvenes», en Educación, convivencia y ciudadanía en la cultura global, coords. Javier Marrero Acosta y Javier Argos González (Las Rozas: Wolters Kluwer, 2008), 177-195; «Deshaciendo equívocos: la Educación para la Ciudadanía en el contexto europeo», Idea La Mancha. Revista de Educación de Castilla-La Mancha, 6 (2008): 79-84.
} 
para mantener otros, que cuando todo el debate se centra en un solo asunto, en relación con el cual no puede haber más que ganadores y perdedores. La posibilidad de llevar a cabo procesos en los cuales todos ganen y pierdan algo contribuye en buena medida al éxito de la negociación. En realidad, más que conseguir unos acuerdos que respondan completamente a ciertas posiciones y descarten totalmente otras, el objetivo debería ser conseguir acuerdos que agraden o desagraden a todos por igual, de manera equilibrada. Y por eso los procesos globales de discusión, que abarcan varios elementos, son más aconsejables para obtener buenos resultados en la negociación.

Estoy convencido de que eso es lo que sucedió con la LOE. Pasado el tiempo he escuchado numerosas valoraciones, que suelen incluir discrepancias con tal o cual aspecto concreto del texto aprobado. Pero es difícil encontrar posiciones absolutamente a favor o en contra de la ley. En eso consistió su aceptación, que creo que fue bastante generalizada, realidad que contrasta frontalmente con la experiencia de la LOMCE, en la que hubo sectores victoriosos y otros claramente agraviados.

El 6 de abril de 2006 finalmente se aprobó la LOE en el Parlamento. La ley fue sancionada por el rey Juan Carlos I el 3 de mayo y publicada en el Boletín Oficial del Estado del día 4 de mayo. Terminaba así el proceso de reversión de la LOMCE abierto dos años antes, dentro del plazo previsto y con los resultados apetecidos.

A partir de aquí tendría lugar el proceso de desarrollo de la ley, que incluyó la publicación de los reales decretos que regulaban la educación infantil, primaria, secundaria obligatoria y bachillerato, ${ }^{21}$ más un buen número de órdenes ministeriales. Por su parte, las comunidades autónomas fueron publicando sus normas reguladoras del currículo de las diferentes etapas y de la aplicación de la ley. Fue un proceso intenso y que llevó un tiempo considerable, pero que respondió a una planificación cuidadosa y a procesos sostenidos de negociación con las organizaciones educativas y con las comunidades autónomas. No me centro aquí en esta etapa, que desborda mis

\footnotetext{
${ }^{21}$ Real Decreto 1630/2006, de 29 de diciembre, por el que se establecen las enseñanzas mínimas del segundo ciclo de Educación infantil (BOE 4 enero 2007); Real Decreto 1513/2006, de 7 de diciembre, por el que se establecen las enseñanzas mínimas de la Educación primaria (BOE 8 diciembre 2006); Real Decreto 1631/2006, de 29 de diciembre, por el que se establecen las enseñanzas mínimas correspondientes a la Educación Secundaria Obligatoria (BOE 5 enero 2007); Real Decreto 1467/2007, de 2 de noviembre, por el que se establece la estructura del bachillerato y se fijan sus enseñanzas mínimas (BOE 6 noviembre 2007).
} 
objetivos concretos para este trabajo, pero no quiero dejar de subrayar la importancia que tuvo una buena planificación de esta fase, incluso antes de cerrar la aprobación de la ley matriz, así como de continuar manteniendo un proceso de trabajo conjunto y una negociación permanente, con el fin de evitar resistencias y llamadas a la falta de aplicación, como está sucediendo en la actualidad con la LOMCE.

\section{UNA REFLEXIÓN FINAL SOBRE EL PAPEL DE LO ALEATORIO EN LA CONSTRUCCIÓN DE POLÍTICAS}

Hasta aquí he venido presentando el proceso que llevó a plantear la necesidad de elaborar una nueva ley general de educación en las circunstancias políticas que se vivían en el año 2004, el modo en que tuvo lugar su traducción en decisiones de actuación, la construcción de una estrategia basada en el diálogo y la búsqueda de consensos para la elaboración de la ley, las negociaciones mantenidas dentro y fuera del marco parlamentario y la aprobación final de la LOE. Espero que dicha presentación haya sido clarificadora y que las reflexiones que la han ido acompañando hayan resultado pertinentes e interesantes. Pero hay un asunto que desde que viví aquella experiencia no ha dejado de inquietarme y sobre el cual querría llamar la atención. Se trata del papel relevante, en ocasiones decisivo, que desempeña el azar en la construcción de políticas.

En efecto, de la descripción realizada pudiera inferirse que todo el proceso de elaboración y aprobación de la LOE siguió un itinerario guiado por la racionalidad, la planificación y el cálculo. Es cierto que, acostumbrado el grupo promotor al trabajo académico, adoptamos esos criterios como guía para nuestra actuación. Pero sin embargo, hay que reconocer que la realidad así presentada no deja de ser parcial, pues falta por mencionar el impacto de lo aleatorio, que en ocasiones obligó incluso a cambiar el curso de los acontecimientos.

Para quien tiene experiencia en la actuación política no supone ningún problema reconocer la importancia de los fenómenos aleatorios, debidos al azar, en el curso de la misma. El hecho de que una noticia se publique en la prensa o se extienda un rumor interesado puede acabar afectando a la argumentación en favor de una medida determinada o incluso puede obligar a tomar otro rumbo de acción. El hecho de que la tensión política aumente o disminuya incide directamente en el debate parlamentario de 
una norma determinada, independientemente de su carácter y su grado de deseabilidad. Y así sucede con otros fenómenos que los promotores de una política determinada no controlan. En las páginas anteriores se encuentran ejemplos claros de esa realidad.

Así, por ejemplo, el hecho de que el mencionado intento de pacto social por la educación, laboriosamente trabajado, discreto y riguroso, que exploraron varias grandes organizaciones educativas, se saldara con el fracaso por una filtración interesada impidió beneficiarse de una plataforma que sin duda hubiese obligado a los grupos políticos a escuchar sus propuestas y tomarlas en consideración, al margen de que decidieran hacerlas finalmente suyas o no. Cabe pensar lo diferente que hubiese podido ser el proceso de elaboración de la LOE y su debate parlamentario si se hubiese alcanzado ese acuerdo en forma de pacto básico.

Otro tanto cabe decir acerca del impacto que tuvo la irrupción del debate territorial asociado a la discusión en torno al Estatuto de Cataluña, que como hemos visto impidió llegar a ningún acuerdo con el PP acerca de la LOE. Quizás si ese fenómeno no hubiese tenido lugar, o simplemente se hubiese planteado tiempo después, el resultado de la ley hubiese podido ser distinto y se hubiese conseguido el efecto indirecto de una mayor estabilidad normativa.

Y también se produjo un efecto inesperado cuando la votación parlamentaria de los artículos de la LOE que regulaban la educación infantil cambió el texto original, acordado además con ERC pese a su discrepancia con el papel que concedían a la administración central, disminuyendo el grado de regulación estatal del primer ciclo y dejando el establecimiento de su currículo y condiciones en manos de las comunidades autónomas. Ese imprevisto obligó a modificar la línea de actuación que el ministerio se había trazado para esa etapa, incluso en contra de nuestras propias convicciones.

Como puede apreciarse, y debo decir que los ejemplos mencionados no constituyen una relación exhaustiva, no todo puede ser previsto en un proceso de construcción de políticas públicas. Hay que estar atentos para dar respuesta a las situaciones inesperadas y azarosas que se plantean, que en ocasiones pueden ser esquivadas y otras veces no y que siempre suelen incidir profundamente en el curso de acción. En consecuencia, tanto en la actuación política como en su análisis académico conviene evitar el riesgo de 
interpretar todo como si fuese el simple resultado de una actuación racional y hay que dejar espacio para la intervención de lo inesperado, lo aleatorio o lo simplemente disruptivo. Me parece esta una reflexión importante, que no me hubiese aventurado a realizar antes de vivir una experiencia personal como la con la que quiero cerrar estas páginas.

\section{Nota sobre el autor:}

Alejandro Tiana Ferrer (Madrid, 1951), licenciado y doctor en Filosofía y Letras (Pedagogía) por la Universidad Complutense de Madrid, es catedrático de la Universidad Nacional de Educación a Distancia (UNED), de la que es actualmente Rector. Ha desarrollado su docencia universitaria desde el año 1980 en las áreas de Historia de los sistemas educativos contemporáneos, Política y legislación educativa, Educación comparada y Evaluación de la educación. Ha desempeñado varias responsabilidades en la UNED, además de ocupar cargos en el Ministerio de Educación y Ciencia de España, como Secretario General de Educación (2004-2008), director del Instituto Nacional de Calidad y Evaluación (1994-1996) y director del Centro de Investigación y Documentación Educativa (1989-1994). Entre 2008 y 2012 fue director del Centro de Altos Estudios Universitarios de la Organización de Estados Iberoamericanos para la Educación, la Ciencia y la Cultura (OEI). Ha trabajado con diversas organizaciones internacionales, como UNESCO, OCDE, Banco Mundial, OEI, Banco Interamericano de Desarrollo y ALECSO y ha sido miembro de comisiones nacionales de Francia, Portugal, Marruecos, Argentina y México. Es autor o coautor de 23 libros y más de 200 artículos o capítulos de libros sobre diversos temas de historia de sus especialidades. 